\title{
Why did we elaborate an entangled photons experiment in our engineering school?
}

Lionel Jacubowiez, Thierry Avignon

Lionel Jacubowiez, Thierry Avignon, "Why did we elaborate an entangled photons experiment in our engineering school?," Proc. SPIE 9664, Ninth International Topical Meeting on Education and Training in Optics and Photonics, 966411 (24 October 2005); doi: 10.1117/12.2207539 
Ref ETOP006

\title{
Why did we elaborate an entangled photons experiment in our engineering school?
}

\author{
Lionel Jacubowiez, Thierry Avignon
}

Institut d'Optique, Ecole Supérieure d'Optique

Centre Universitaire, BP 147, Orsay 91403 France

\begin{abstract}
We will describe a simple setup experiment that allows students to create polarization-entangled photons pairs. These photon pairs are in an entangled state first described in the famous 1935 article in Phys.Rev by Einstein-Podolsky-Rosen, often called E.P.R. state. Photons pairs at $810 \mathrm{~nm}$ are produced in two nonlinear crystals by spontaneous parametric downconversion of photons at $405 \mathrm{~nm}$ emitted by a violet laser diode. The polarization state of the photons pairs is easily tunable with a half-wave plate and a Babinet compensator on the laser diode beam. After having adjusted the polarization-entangled state of the photon pairs, our students can perform a test of Bell's inequalities. They will find the amazing value for the Bell parameter between 2.3 and 2.6, depending on the quality of the adjustments of the state of polarization. The experiments described can be done in 4 or 5 hours.

What is the importance of creating an entangled photons experiment for our engineering students?

First of all, entanglement concept is clearly one of the most strikingly nonclassical features of quantum theory and it is playing an increasing role in present-day physics. But in this paper, we will emphasise the experimental point of view. We will try to explain why we believe that for our students this lab experiment is a unique opportunity to deal with established concepts and experimental techniques on polarization, non linear effects, phase matching, photon counting avalanche photodiodes, counting statistics, coincidences detectors.

Let us recall that the first convincing experimental violations of Bell's inequalities were performed by Alain Aspect and Philippe Grangier with pairs of entangled photons at the Institut d'Optique between 1976 and 1982. Twenty five years later, due to recent advances in laser diode technology, new techniques for generation of photon pairs and avalanche photodiodes, this experiment is now part of the experimental lab courses for our students.
\end{abstract}

\section{Keywords}

Bell inequalities, entangled states, nonlinear optics parametric processes, photon counting, polarization 


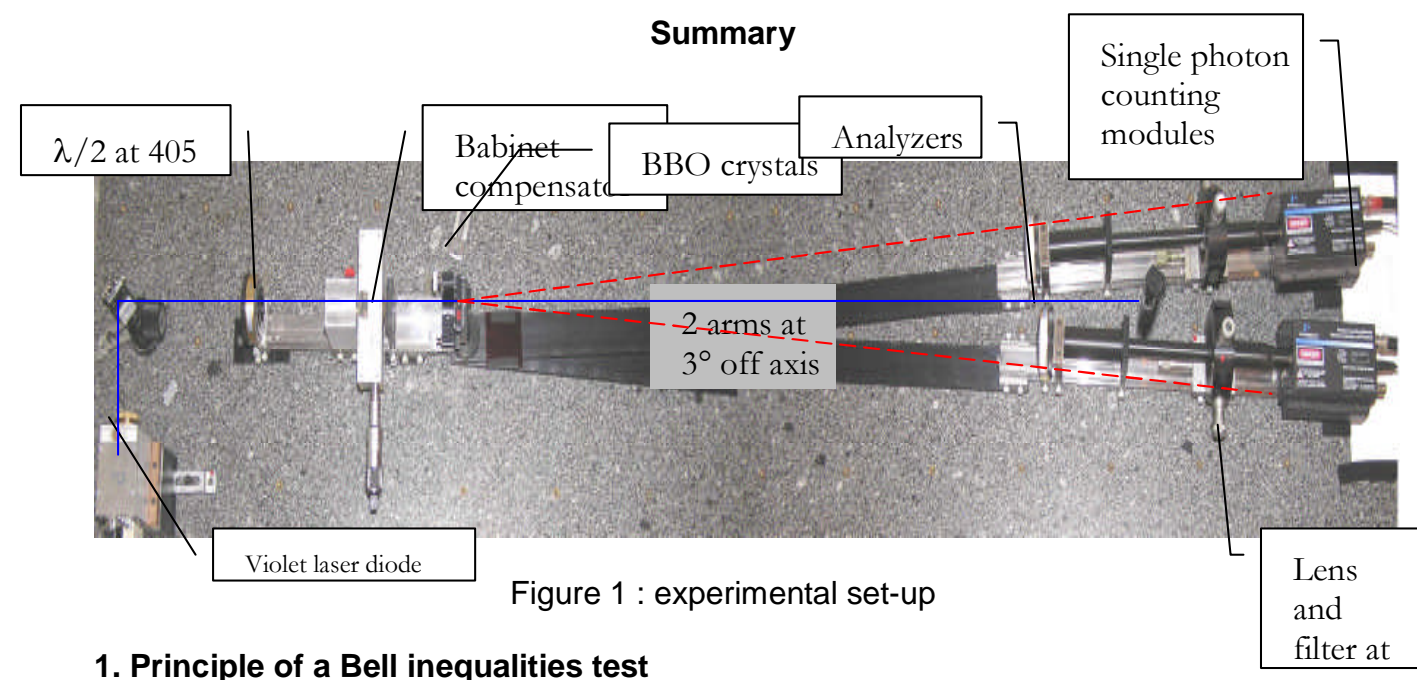

1. Principle of a Bell inequalities test

EPR "gedankenexperiment" with polarization entangled photons, first presented by Bohm, is shown on the next figure. Polarization state of the entangled photon pairs created by the source is:

$|\psi\rangle_{E P R}=\frac{1}{\sqrt{2}}\left(\left|V_{I}\right\rangle\left|V_{I I}\right\rangle+\left|H_{I}\right\rangle\left|H_{I I}\right\rangle\right)$

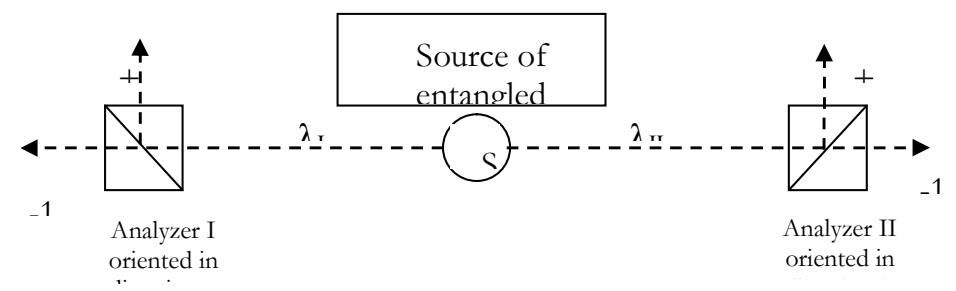

Figure 2 : Einstein-Podolsky-Rosen-Bohm « gedankenexperiment » with photons.

On both sides, each photon is analysed by a polarization beam splitter cube. The result of this measurement is +1 if the photon is detected with a vertical polarization in direction $\mathrm{a}$ (or $\mathrm{b}$ on the other side), or -1 if the photon is detected with a polarization perpendiculare to direction $a$ (or $b$ on the other side). One can measure the probabilities of simple detections or the probabilities of double simultaneaous detections with different orientations of the beam splitter cubes.

The correlations between the results on the polarization tests, for any directions $a$ and $b$ of the analysers can be defined by : 
$E(I a, I I b)=P\left(V_{I a}, V_{I b}\right)+P\left(H_{I a}, H_{I b}\right)-P\left(H_{I a}, V_{I b}\right)-P\left(V_{I a}, H_{I b}\right)$

If there is not any correlation between the two measurements: $E(I \quad a, I I b)=0$.

At the opposite extreme, if $E(I a, I I b)=1$ or -1 , the correlation is perfect.

For an EPR pair of entangled photons, Quantum Mechanics predicts:

$E(I a, I I b)=\cos [2(a-b)]$

Then if you measure the Bell parameter given by :

$S_{B e l l}\left(a, a^{\prime}, b, b^{\prime}\right)=E(I a, I I b)-E\left(I a, I I b^{\prime}\right)+E\left(I a^{\prime}, I I b\right)+E\left(I a^{\prime}, I I b^{\prime}\right)$

with the orientations shown on the next figure, you should obtain $S_{B e l l}\left(\mathrm{a}, \mathrm{a}^{\prime}, \mathrm{b}^{\mathrm{b}}, \mathrm{b}^{\prime}\right)=2 \sqrt{2}$.

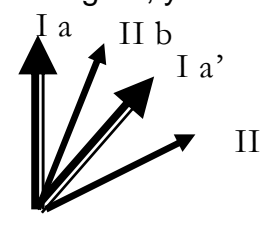

Figure 3: Orientations which give the maximum conflict between Bell inequalities and Quantum Mechanics For $a, b=b, a^{\prime}=a^{\prime}, b=22^{\circ} 5, S_{B e l l}\left(\mathrm{a}, \mathrm{a}^{\prime}, \mathrm{b}, \mathrm{b}^{\prime}\right)=2 \sqrt{2}$ (Q. M.)

Bell famous theorem states that any Local Hidden Variable Theories will give: $-2 \leq S_{\text {Bell }}\left(\mathrm{a}, \mathrm{a}^{\prime}, \mathrm{b}^{\mathrm{b}} \mathrm{b}^{\prime}\right) \leq 2$

With the simple setup experiment that we decided to build in our engeneering school, our third year students can create polarization-entangled photons pairs and perform a test of Bell inequalities in 4 or 5 hours.

\section{The source of polarization-entangled photon pairs}

Entangled photons are created by type I spontaneous parametric downconversion in BBO ( $\square$-Borate de Baryum) non linear crystals. The pump, a $60 \mathrm{~mW}$ at $405 \mathrm{~nm}$ blue diode, gives $810 \mathrm{~nm}$ entangled photons.

We use two crystals, $5 \mathrm{~mm} \times 5 \mathrm{~mm} 0.5 \mathrm{~mm}$ thick) cut with their crystal axis at $30^{\circ}$ from normal to the large face. For this angle the indexes are:
ne à $405 \mathrm{~nm}=$
1.65777
ne à $810 \mathrm{~nm}=1.62854$
no à $810 \mathrm{~nm}=1.65998$
no à $405 \mathrm{~nm}=1.69172$

In a downconversion process, energy and photon momentum are conserved:

$\frac{h c}{\lambda_{p}}=\frac{h c}{\lambda_{s}}+\frac{h c}{\lambda_{i}}$
$\frac{n_{e}}{\lambda_{p}} \vec{u}_{p}=\frac{n_{o}}{\lambda_{s}} \vec{u}_{s}+\frac{n_{o}}{\lambda_{i}} \vec{u}_{i}$

With these indexes, we expect type I phase matching, which means that an extraordinary polarized pump photon will create two ordinary polarised "daughter photons". 
We select "degenerate" daughter photons, those which have the same wavelength: $\lambda_{\text {signal }}=\lambda_{\text {iddler }}=2 \lambda_{\text {pump }}$.

So :

$\frac{n_{e}}{\lambda_{405 n m}} \vec{u}_{\text {pompe }}=2 \frac{n_{0}}{\lambda_{810 n m}} \cos \left(\vec{u}_{s}, \vec{u}_{p}\right)$

$\left(\vec{u}_{s}, \vec{u}_{p}\right)=\cos ^{-1}\left(\frac{n_{e}}{n_{0}}\right)=2^{\circ} 57^{\prime}$

Degenerated Photons pairs are emitted in a cone of angle about $3^{\circ}$ around the pump direction.

To produce polarization entangled pairs of photons, we use two identical crystals, rotated by $90^{\circ}$ from each other about the pump beam direction. In this configuration, $45^{\circ}$ polarized pump photons can downconvert in either crystal (but it is not possible to know in which crystal the photon pairs were created!).

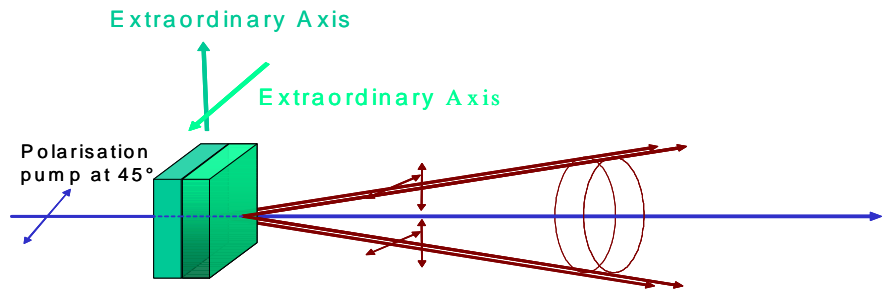

Figure 3 : two crystals downconversion source

\section{Detection of the polarization-entangled photon pairs}

Downconverted photons are collected by two lenses (focal : $75 \mathrm{~mm}$, diameter : $10 \mathrm{~mm}$ ) at about one meter from the crystals and focussed on single photon counting avalanche photodiodes.

Filters at $810 \mathrm{~nm}, 10 \mathrm{~nm}$ width, are placed just in front of each lens. Polarization is analysed by rotating the half wave plates in front of the polarization beam splitter cubes. Black plastic tubes prevent from stray light and protect single photon counting modules. For each detected single photon these modules give a 25 ns TTL pulse which is sent to a simple coincidence detector. The coincidence window is $30 \mathrm{~ns}$ width. We have 3 counters to measure both single detection rates and the coincidence rate.

In our experiment, for single detection rates of about 23000 on each side, we detect about 1600 coincidences per second.

\section{Adjustment of the EPR polarization state}

For a rectilinearly polarised pump at $45^{\circ}$, the pump photons state is $|\psi\rangle_{\text {pump }}=\frac{1}{\sqrt{2}}(|V\rangle+|H\rangle)$ and two downconversion processes are equally probable:

$\left\{\right.$ Downconversion in the vertical crystal : $|V\rangle_{\text {pump }} \rightarrow e^{i \phi_{1}}\left|H_{I}\right\rangle\left|H_{I I}\right\rangle$

Downconversion in the horizontal crystal : $|H\rangle_{\text {pump }} \rightarrow e^{i \phi_{2}}\left|V_{I}\right\rangle\left|V_{I I}\right\rangle$ 
Thus the photon pairs are in the superposition state:
$|\psi\rangle_{\text {photon pair }}=\frac{1}{\sqrt{2}}\left(\left|V_{I}\right\rangle\left|V_{I I}\right\rangle+e^{i \phi}\left|H_{I}\right\rangle\left|H_{I I}\right\rangle\right)$ where $\square=\square \tilde{G} \square$ is a the phase which depends on many different parameters (wavelengths of pump and downconverted photons, for example).

This state leads to a detection probability of coincidence for both analysers oriented at

$45^{\circ}$ :

$$
P\left(V_{I 45^{\circ}}, V_{I I 45^{\circ}}\right)=\frac{1}{4}(1+\cos \phi)
$$

If $\square=0$, entangled photons are actually in the EPR state as defined before .

If $\square=\square P\left(V_{I 45^{\circ}}, V_{I I 45^{\circ}}\right)=0$, entangled photons are in the state : $|\psi\rangle_{E P R}=\frac{1}{\sqrt{2}}\left(\left|V_{I}\right\rangle\left|V_{I I}\right\rangle-\left|H_{I}\right\rangle\left|H_{I I}\right\rangle\right)$

If. $\square=\tilde{\square} \square$ there is not any correlation between the polarization measurements in the diagonal base :

$P\left(V_{I 45^{\circ}}, V_{I I 45^{\circ}}\right)=1 / 4$

To perform a Bell test, it is very important to adjust precisely this phase between the two possible dowconversion processes.

We have decided to control this phase by a Babinet compensator on pump beam. It is possible to measure $P\left(V_{I 45^{\circ}}, V_{I I 45^{\circ}}\right)$ versus the position of the Babinet compensator when both analysers are oriented at $45^{\circ}$. Thus, we adjust precisely the Babinet to obtain a maximum number of coincidences per second in this configuration

\section{Bell's Parameter Measurement}

The Bell parameter is obtained by the measurements of 4 coefficients of correlation, $E\left(V_{a}, V_{b}\right)$.

$S_{\text {Bell }}\left(\mathrm{a}, \mathrm{a}^{\prime}, \mathrm{b}, \mathrm{b}^{\prime}\right)=E\left(V_{a}, V_{b}\right)-E\left(V_{a}, V_{b^{\prime}}\right)+E\left(V_{a^{\prime}}, V_{b}\right)+E\left(V_{a^{\prime}}, V_{b^{\prime}}\right)$

Each of these coefficients needs 4 coincidence rate measurements, $\mathrm{n}(\mathrm{a}, \mathrm{b})$.

$n_{\text {total }}=n(a, b)+n(a+90, b+90)+n(a, b+90)+n(a+90, b)$

$P\left(V_{a}, V_{b}\right)=n(a, b) / n_{\text {total }}$

$P\left(H_{a}, H_{b}\right)=n(a+90, b+90) / n_{\text {tot }}$

$P\left(V_{a}, H_{b}\right)=n(a, b+90) / n_{t o t}$

$P\left(H_{a}, V_{b}\right)=n(a+90, b) / n_{t o t}$

$E\left(V_{a}, V_{b}\right)=\frac{n(a, b)+n(a+90, b+90)-n(a, b+90)-n(a+90, b)}{n(a, b)+n(a+90, b+90)+n(a, b+90)+n(a+90, b)}$

In our experiment, we measure these coincidence rates in 15 seconds each. We obtain $S_{\text {Bell }}\left(\mathrm{a}, \mathrm{a}^{\prime}, \mathrm{b}^{\mathrm{b}} \mathrm{b}^{\prime}\right)=2,48$ with a standard deviation $\square=3.10-3$. This result is in complete disagreement with any hidden variable local theory.

\section{Short Conclusion}

Since the eighties, the EPR paradox is no longer a "gedankenexperiment". Now, it has become a very exciting lab work for students. We believe it is enlightening not only for quantum mechanics knowledge but also in many different fields of modern physics. 